\title{
Analysis on the Effect of Early Screening and Safe Management of Dysphagia in Scleroderma Patients
}

\author{
Yingfeng Huang ${ }^{1}$, Guiyan Wen ${ }^{1,}$, Xia Huang ${ }^{1}$, Xiaozhen Huang ${ }^{2}$ \\ ${ }^{1}$ Department of Gastroenterology and Rheumatology, The First Affiliated Hospital of Jinan University, Guangzhou, China \\ ${ }^{2}$ Fever Clinic, The First Affiliated Hospital of Jinan University, Guangzhou, China
}

Email address:

26024497@qq.com (Yingfeng Huang),wengy78@126.com (Guiyan Wen), 15915772676@qq.com (Xia Huang),

183890905@qq.com (Xiaozhen Huang)

${ }^{*}$ Corresponding author

\section{To cite this article:}

Yingfeng Huang, Guiyan Wen, Xia Huang, Xiaozhen Huang. Analysis on the Effect of Early Screening and Safe Management of Dysphagia in Scleroderma Patients. American Journal of Nursing Science. Vol. 9, No. 6, 2020, pp. 442-444. doi: 10.11648/j.ajns.20200906.20

Received: November 25, 2020; Accepted: December 4, 2020; Published: December 11, 2020

\begin{abstract}
Objective: To analyze the effect of early screening and safe management of dysphagia in scleroderma patients. Methods: From January 2016 to January 2020, a randomized controlled trial was performed with scleroderma patients $(n=42)$ who were diagnosed as scleroderma. In intervention group, the participants are accepted early screening and safe management of swallowing disorders. The participants of control group are accepted common nursing service. Result: the $48.84 \%$ of participants have problems in swallowing process, most participants provide assessment of level 2 of WST. In swallowing test assessment, more participants think they feel obstruction when eating steamed bread. The accident aspiration pneumonia has higher risk than that of aspiration pneumonia in two group ( $10.00 \%$ vs $3.75 \% \& 37.50 \%$ vs $23.75 \%)$. In research of swallowing function treatment, most participants of intervention group are basic recovery in treatment outcome, the control group have most invalid assessment in treatment outcome. Conclusion: the early screening and safe management of dysphagia can efficacy improve scleroderma dysphagia of scleroderma patients. In dysphagia problem, the scleroderma patients have higher risk of accident aspiration, and the early screening and safe management of dysphagia can improve problems of accident aspiration and aspiration pneumonia. Additionally, the early screening and safe management of dysphagia have strong effect for dysphagia.
\end{abstract}

Keywords: Scleroderma, Dysphagia, Nursing Intervention

\section{Introduction}

Scleroderma is a connective tissue disorder from autoimmune inflammatory, it results in the sclerosis, or fibrous hardening, of the skin and sometimes the internal organs [1]. Additionally, the patient's blood vessels may also be affected in the systemic scleroderma, the scleroderma lead to endothelial injury resulting in microangiopathy [2]. It has 3 pathogenesis, that includes skin tightening, small blood vessel involvement, and collagen overproduction in various internal organs [3]. Base on esophageal manometry report, nearly $90 \%$ of scleroderma patients presented with reduced esophageal sphincter tension and/or significantly lower esophageal creep. Abnormal gastrointestinal motility can lead to dysphagia, gastroesophageal reflux, satiety, pseudo obstruction [4].

The dysphagia was defined as a difficulty swallowing foods and/or liquid or to the sensation that foods and/or liquids become obstructed on their transit from the mouth to the stomach [5]. In the most common consequences of dysphagia, the patients have many serious problems, that include malnutrition, dehydration, recurrent cough, aspiration pneumonia, choking, limited quality of life, social isolation, increased mortality and morbidity rate $[6,7]$. The scleroderma patients suffer higher risk as they have dysphagia, they are easier to experience complications such as malnutrition, dehydration or aspiration pneumonia, they need more residential care setting [8, 9]. The aim of this study was analyzing on the effect of early screening and safe management of dysphagia in scleroderma patients. 


\section{Methods}

\subsection{Participants Enrollment and Survey Methods}

From January 2016 to January 2020, a randomized controlled trial was performed with scleroderma patients $(n=42)$ who were diagnosed as scleroderma. The patients were regularly seen at rheumatology and immunology department of the First Affiliated Hospital of Jinan University, and their diagnosis according to the Chinese diagnostic criteria for scleroderma [10]. 42 patients were randomly divided into intervention groups and control group, the participants of two groups are accept water swallow test (WST) in study. In intervention group, the participants are accepted early screening and safe management of swallowing disorders. The participants of control group are accepted common nursing service.

WST is a cost-effective bedside screening tool to detect dysphagia in clinical practice $[11,12]$. Base on the report, sensitivity of WST for aspiration ranged from 34.8 to $55.7 \%$, its specificity ranged from 78.9 to $93.2 \%$. Our study assesses participants based on from $2 \mathrm{~mL}$ of water swallowing to $30 \mathrm{~mL}$ of water swallowing.

In safe management of dysphagia, the intervention contrasts related health education and safe eating management. The investigators explained to the patients and their families the influence of abnormal swallowing function on the safety of eating and the methods and importance of preventing the occurrence of aspiration. In addition, we not only provide guidelines and standards for safe eating to patients but also provide safe eating care for patients. The safe eating care include: (1) Make sure the patient has no loose teeth before eating; (2) Compensatory methods of changing postures and postures; (3) Choose the right foods which have suitable quality and character; (4) Assess eating food safety.

\subsection{Statistical Analysis}

Our data analyzer performed the statistical analysis by SPSS 22.0. The $\mathrm{P}$ value, $\mathrm{t}$-test and chi-square test were associated with collection result were analyzed. Besides, the mean standard deviation for statistical description.

\section{Result}

Table 1. Results of swallowing function screening and feeding assessment result.

\begin{tabular}{lll}
\hline Abnormal swallowing results & Cases (n) & Percent (\%) \\
\hline Level 2 of WST & 8 & 18.60 \\
Level 3 of WST & 6 & 13.95 \\
Level 4-5 of WST & 2 & 4.6 \\
Having difficulty swallowing steamed bread & 2 & 4.6 \\
feeling obstruction when eating steamed bread & 3 & 6.5 \\
Total & 21 & 48.84 \\
\hline
\end{tabular}

WST $=$ water swallow test

In Table 1, it shows results of swallowing function screening and feeding assessment result, they include assessment result of level 2-5 of WST and swallowing test assessment. In total, the $48.84 \%$ of participants have problems in swallowing process, most participants provide assessment of level 2 of WST. In swallowing test assessment, more participants think they feel obstruction when eating steamed bread.

We make the statistics and analysis to assess accident aspiration and aspiration pneumonia between the intervention group and control group (Table 2). The accident aspiration pneumonia has higher risk than that of aspiration pneumonia in two group ( $10.00 \%$ vs $3.75 \% \& 37.50 \%$ vs $23.75 \%)$. In addition, the risk of accident aspiration pneumonia and risk of aspiration pneumonia in intervention group are much less than control group.

Table 2. The incidence of accident aspiration and aspiration pneumonia was compared between the two groups.

\begin{tabular}{lll}
\hline Projects & $\begin{array}{l}\text { Accident aspiration } \\
\text { pneumonia }\end{array}$ & $\begin{array}{l}\text { aspiration } \\
\text { pneumonia }\end{array}$ \\
\hline Intervention group $(\mathrm{n}=21)$ & $10.00 \%$ & $3.75 \%$ \\
Control group $(\mathrm{n}=21)$ & $37.50 \%$ & $23.75 \%$ \\
\hline
\end{tabular}

In Table 3, it indicates the result of effect of swallowing function treatment in 2 groups, it contrasts 3 levels assessment. Compare with intervention group and control group, most participants of intervention group are basic recovery in treatment outcome, the control group have most invalid assessment in treatment outcome.

Table 3. The effect of swallowing function treatment in 2 groups was compared.

\begin{tabular}{llll}
\hline Projects & Basic recovery & effective & invalid \\
\hline Intervention group $(\mathrm{n}=21)$ & $38.50 \%$ & $18.00 \%$ & $5 \%$ \\
Control group $(\mathrm{n}=21)$ & $17.50 \%$ & $21.25 \%$ & $41.25 \%$ \\
\hline
\end{tabular}

\section{Discussion}

Scleroderma is an autoimmune rheumatic disease, the patients suffer substantial damage of vascular system, tissue fibrosis and accumulation of collagen in skin [13]. it is a disease in which hardening of skin and connective tissue occurs either locally or all over the body. In scleroderma, scleroderma has been classified into localized and systemic [14]. Despite of the details of its pathogenesis remain unclear, its characteristics are pro-inflammatory cytokines, autoimmunity, and high levels of autoantibodies contribute to micro-vascular damage, inflammation, and fibrosis [2, 15]. The dysphagia is a potential safety problem in scleroderma, the patients have risk of choking to death while eating. We not only identified the biggest risk factors for swallowing problems, but also evaluated the role of early screening and safe management of dysphagia in swallowing problems.

According to the above results, the early screening and safe management of dysphagia can efficacy improve scleroderma dysphagia of scleroderma patients. Base on Table 1, the $48.84 \%$ of scleroderma patients have dysphagia problems, it means that the most scleroderma patients are difficult to eat in their life, it also is a serious clinical problem. The Table 2 indicates the dysphagia problems which from more scleroderma patients are accident aspiration, not aspiration pneumonia. In addition, the participants who receipt early screening services 
and safe management of dysphagia are safety in dysphagia, they have lower risk than that of participants who receipt common intervention service. According to the result of swallowing function treatment, the intervention group participants have better outcome of swallowing function treatment than that of control group participants. Thus, the early screening and safe management of dysphagia have good effect for swallowing of scleroderma patients. In similar, $\mathrm{Hu}$ had similarly research result for our study, that early screening service can improve the dysphagia problems of scleroderma patients. But he did not use safe management of dysphagia to improve swallowing function of scleroderma patients [16].

\section{Conclusion}

In conclusion, the early screening and safe management of dysphagia can efficacy improve scleroderma dysphagia of scleroderma patients. In dysphagia problem, the scleroderma patients have higher risk of accident aspiration, and the early screening and safe management of dysphagia can improve problems of accident aspiration and aspiration pneumonia. Additionally, the early screening and safe management of dysphagia have strong effect for dysphagia.

\section{References}

[1] Alhajeri H, Hudson M, Fritzler M, Pope J, Tatibouet S, Markland J, et al. 2013 American College of Rheumatology/European League against rheumatism classification criteria for systemic sclerosis outperform the 1980 criteria: data from the Canadian Scleroderma Research Group. Arthritis Care Res. 2015; 67 (4): 582-587.

[2] Peytrignet S, Denton CP, Lunt M, Hesse strand R, Mouthon L, Silman A, Pan, X, et al. Disability, fatigue, pain and their associates in early diffuse cutaneous systemic sclerosis: the European scleroderma observational study. Rheumatology. 2018; 57 (2): 370-381.

[3] Siegert E, March C, Otten L, Makowka A, Preis E, Buttgereit F, et al. Prevalence of sarcopenia in systemic sclerosis: assessing body composition and functional disability in patients with systemic sclerosis. Nutrition. 2018: 55-56, 51-55.

[4] Xu W, Zhang MJ. Progress in clinical manifestations, diagnosis and treatment of scleroderma digestive system involvement Chinese Clinical Medicine August. 2016; 23 (4): 519-524.

[5] Malagelada JR, Bazzoli F, Boeckxstaens G, De Looze D, Fried M, Kahrilas P, Lindberg G, Malfertheiner P, Salis G, Sharma P, Sifrim D, Vakil N, Le Mair A. World Gastroenterology
Organisation Global Guidelines: Dysphagia - Global Guidelines and Cascades Update September 2014. Journal of Clinical Gastroenterology. 2015; 49 (5): 370-378.

[6] Baijens LWJ, Clavé P, Cras P, Ekberg O, Forster A, Kolb GF, Leners JC, Masiero S, Mateos-Nozal J, Ortega O, Smithard DG, Speyer R, Walshe M. European Society for Swallowing Disorders - European Union Geriatric Medicine Society white paper: oropharyngeal dysphagia as a geriatric syndrome. Clinical Interventions in Aging. 2016; 11: 1403-1428.

[7] Namasivayam AM, Steele CM. Malnutrition and dysphagia in long term care: A systematic review. Journal of Nutrition in Gerontology and Geriatrics. 2015; 34 (1): 1-21.

[8] Ekberg O, Hamdy S, Woisard V, Wuttge-Hannig A, Ortega P. Social and psychological burden of dysphagia: Its impact on diagnosis and treatment. Dysphagia. 2018; 17 (2): 139-146.

[9] Cohen D, Roffe C, Beavan J, et al. Post-stroke dysphagia: a review and design con-side rations for future trials. Int J Stroke. 2016: 1-13.

[10] Cui XX, Yang J, Tu WZ, LI M. Cutaneous manifestations and their clinical significance in patients with systemic sclerosis. Chinese Journal of Dermatology. 2016; 51 (01): 14-19.

[11] Bours GJ, Speyer R, Lemmens J, Limburg M, de Wit R. Bedside screening tests vs. video fluoroscopy or fibreoptic endoscopic evaluation of swallowing to detect dysphagia in patients with neurological disorders: systematic review. J Adv Nurs. 2009; 65 (3): 477-493.

[12] Chen PC, Chuang CH, Leong CP, Guo SE, Hsin YJ. Systematic review and meta-analysis of the diagnostic accuracy of the water swallow test for screening aspiration in stroke patients. J Adv Nurs. 2016; 72: 2575-86.

[13] Wang Z, Yin X, Gao L, Feng S, Song K, Li L. The protective effect of hydrogen sulfide on systemic sclerosis associated skin and lung fibrosis in mice model. Springer plus. 2016; 5: 1084.

[14] Koca SS, Ozgen M, Dagli AF, Gozel N, Ozercan IH, Isik A. The protective effects of Bevacizumab in bleomycin-induced experimental scleroderma. Adv. Clin. Exp. Med. 2016; 2: 249253.

[15] Knobler R, Moinzadeh P, Hunzelmann N, et al. European dermatology forum S1-guideline on the diagnosis and treatment of sclerosing diseases of the skin, Part 2: scleromyxedema, scleredema and nephrogenic systemic fibrosis. J Eur Acad Dermatol Venereol: JEADV. 2017; 31 (10): 1581-1594.

[16] Zhiwei H, Jimin W, Zhonghao W, Yu Z, Tao J, Chao Y, Xing D. Individualized treatment of severe gastroesophageal reflux disease caused by scleroderma: case and literature analysis $[\mathrm{J}]$. Chinese journal of basic and clinical sciences. 2016; 23 (10): 1214-1219. 